führend namhaft machen konnte. Es hat lange gedauert, bis die hierdurch angedeutete sehr weite Verbreitung des Calciumoxalates grössere Beachtung fand, denn von einzelnen fruthern Angaben abgesehen, sind eigentlich erst Mey en 's Erörterungen (1828 - 1837) für die Oxalatkrystalle von allgemeinerer Bedeutung. ${ }^{1}$

(Schluss folgt.)

\title{
Ueber einige Wasserflltrirapparate.
}

Von Dr. Alb. Link, Korps-Stabsapotheker.

In neuerer Zeit habe ich einige der zur Verbesserung des Wassers empfohlenen Wasserfiltrirapparate bezüglich ihrer Leistungsfähigkeit einer Prüfung unterzogen. Die einschlägigen Versuche und Beobachtungen dürften für manche Kreise nicht ohne Interesse sein, weshalb ich dieselben hier folgen lasse.

Zur Prüfung gelangten 3 solcher Filtrirapparate und zwar

1) ein von der Firma G. Arnold und Schirmer in Berlin fabricirtes Patent-Schnellfilter, System Piefko,

2) oin Buhring'sches Kohlefilter, von der Firma C. Bühring und Cie. in Hamburg und

3) ein von dem Ingenieur Breyer in Wien construirtes Mikromembranfilter.

Die Construction der ersten 2 Filter kann wohl als bekannt vorausgesetzt werden. Ich beschränke mich daher an dieser Stelle auf den Hinweis, dass boi dem Piefke'schen Patent-Schnellfilter als Filtermasse Cellulose dient, welche mit Wasser zu einem zarten Brei vertheilt und in dieser Form auf die Siebböden der Filterkammern gebracht wird und dass das Bühring'sche Kohlefilter als Filtermasse porőse Koble und Knochenkohle enthält. Weniger bekannt dürfte das Mikromembranfilter sein, dessen Construction ich daher in allgemeinen Zügen wiedergebe. Ausfthhrlichere Mittheilungen hieruber bringt die Brochüre des Erfinders des Filters "Das Mikromembranfilter. Ein neues technisches Hilfsmittel zur Gewinnung von pilzfreiem Wasser etc." 3. Auflage. Von Friedrich Breyer, Wien 1885, auf welche ich dieserhalb vorweise. Die Filtermasse besteht bei diesem Filter aus Asbest, welcher, nach den in der erwähnten Brochüre enthaltenen Angaben, nach vorgängiger Zerkleinerung bis

1) Vergl. weiter Holzner, tber die Krystalle in Pflanzenzellen. Flora 1862. 499 . 
zur Wollform in nassem Zustando mit krystallinischem kohlensauren Kalk auf einer eigenthtlmlich construirten Mühle höchst fein zermahlen, hierauf mit Salzsäure entkalkt und nach dem Auswaschen der Salzaãure auf einer Mullunterlage in Papierform niedergeschlagen wird. .Die so erhaltenen Filterlamellen sind ihrer äusseren Beschaffenheit nach dickem Filtrirpapier sehr ähnlich; in trockenem Zustande besitzen sie einen ziemlich hohen Grad ron Festigkeit, der durch Befeuchten mit Wasser vermindert wird. Diese Filterlamellen, von Breyer "mobile Mikromembranlamellen" genannt, werden auf dio beidon Seiten von tafelformigen Metallrosten, welche an den Seitenflächen mit vernickelton Messinggewoben überzogen sind und ausserdem je 2 von innen nach aussen gehende Abflussröhrchen enthalten, mittelst einer alkoholischen Schellacklösung unter sorgfältiger Abdichtung des Randes befestigt. Das nunmehr fertige "Doppelmembranelement" kann nach Verschluss des einen und Verbindung des anderen Abflussrölurchens mit einem Abflussschlauch direct als Filter kleinster Art, als ,Taschenfilter", Verwendung finden, oder aber es werden beliebig viele dieser Membrandoppelelemente unter Verbindung der Auslauferöhrchen zu "Elomentbatterien" veroinigt. Boi der Filtration passirt das zu filtrirende Wasser den Apparat von aussen nach innen uud der Abfluss des filtrirten Wassers erfolgt durch die Auslauferöhrchen.

Die mit den 3 Filtrirapparaten angestellten Beobachtungen erstreckten sich

a. auf das Reinigungsvermögen der Filter hinsichtlich der gröberen mechanischen Verunreinigungen des Wassers,

b. auf die Veränderung des Gohalts an Mikroorganismon und

c. auf die Wirkung der Filter in chemischer Beziohung.

I. Versuche mit dem Patent-Schnellfilter von Schirmer und Arnold, System Piefke.

Hiesiges Leitungswasser, welches durch Sandfilter filtrirtes Oderwasser ist und durch diesen Filtrirprocess zwar ziemlich klar erhalten wird, aber immerhin noch kleine Fasern etc. suspendirt enthält, sowie schwach grünlich gefärbt ist, wurde vermittelst des Apparates filtrirt. Das Wasser wurde hierdurch nicht klarer und der schwach grunliche Farbton nicht beseitigt. Nunmehr wurde Oderwasser, welches durch zahlreiche Beimengungen stark getrübt war, der Filtration unterzogen. Das Resultat war eine wesentliche Verminderung der Trubungen, 
ohne dass indess das Wasser, selbst nach wiederholter Filtration, eine ähnliche Klarheit wie das hiesige Leitungswasser erreichte. Vor der Filtration enthielt das Wasser pro ccm 54600 und nach der Filtration 54200 auf Koch'scher Nährgelatine zur Entwicklung gelangende Mikroorganismen. - Ein weiterer Versuch mit sehr trübem, flockigem Teichwasser lieferto ein zwar weniger stark, aber immerhin noch deutlich trübes Filtrat, der Gohalt an Mikroorganismen betrug pro ccm Wasser vor der Filtration 23000 und nach der Filtration 25000. - Nach dem wenig günstigen Ausfall dieser Versuche war es nicht sonderlich wahrscheinlich, dass das Filter im Stande sein würde, ein durch fein vertheilten Thon getrübtes Wassor (eine annähernde Nachahmung der in natürlichen Wässern manchmal vorlommenden Thontrübungen) nennenswerth zu reinigen. Ein Versuch lestätigte diese Voraussetzung, das Filtrat blieb stark durch Thon getrübt. Schliesslich gelangten noch Wasserproben zur Untersuchung, welche durch einen im praktischen Gebrauch befindlichen Apparat gleicher Construction filtrirt worden waren. Das nicht filtrirte Wasser (cin Teichwasser) war milchig getrübt und enthielt zahlreiche Algen. In dem filtrirten Wasser war eine Abnahme dieser Trübungen nicht zu bemerken. Die chomische und bakterioscopische Untersuchung beider Wasserproben ergab folgendes Resultat:

\begin{tabular}{|c|c|c|c|c|c|c|c|}
\hline & \multirow[t]{2}{*}{$\begin{array}{l}\text { Härto- } \\
\text { grade, } \\
\text { deut- } \\
\text { sche }\end{array}$} & $\mid \begin{array}{c}\text { Redu- } \\
\text { cirtes } \\
\text { Kalium- } \\
\text { perman- } \\
\text { ganat }\end{array}$ & $\begin{array}{l}\text { Sal- } \\
\text { poter- } \\
\text { säure }\end{array}$ & $\begin{array}{l}\text { Sal- } \\
\text { petrige } \\
\text { Säure }\end{array}$ & $\begin{array}{c}\text { Ammo- } \\
\text { niak }\end{array}$ & \multirow[t]{2}{*}{ Chlor } & \multirow[t]{2}{*}{$\begin{array}{l}\text { Zur Ent- } \\
\text { wicklung } \\
\text { galangto } \\
\text { Mikroorga- } \\
\text { nismen } \\
\text { nro } 1 \text { ccm } \\
\text { Wasser. }\end{array}$} \\
\hline & & \multicolumn{4}{|c|}{ in 100000 Theilen } & & \\
\hline $\begin{array}{l}\text { Nicht filtrirtes } \\
\text { Wasser }\end{array}$ & 8,8 & 3,48 & 0 & 0 & Spur & 355 & 220 \\
\hline filtrirtos W asser & 8,9 & 3,58 & 0 & 0 & Spur & 3,55 & 1170 \\
\hline Nicht filtrirtos & & & & & & & \\
\hline B. Wasser & 6,6 & 4,59 & 0 & 0 & Spur & 2,91 & 820 \\
\hline filtrirtos Wasser & 6,4 & 4,61 & 0 & 0 & Spur & 2,91 & 5150 \\
\hline
\end{tabular}

Vorstehenden Beobachtungen zufolge ist das Piefke'sche PatentSchnellfilter nicht im Stande, ein durch feinero mechanische Beimengungen getrubtes Wasser von diesen Trubungen $\mathrm{zu}$ befreien, in chemischer Beziehung ist der Apparat ohne jede Wirkung und den Gehalt an Mikroorganismen vermag er nicht herabzusetzen. Es scheint vielmehr, wie die Vorsuche mit dem im praktischen Gebrauch befind- 
lichon Apparato ergeben, dass beim Stagniren des Wassers in dem Apparate eine ziemlich erhebliche Vermehrung der Mikroorganismen vor sich geht, welch letztere ja auch, abgesehen von den anderweit vorhandenen Nahrstoffen, in der Cellùose ein recht glinstiges Nährmaterial vorfinden.

Verkennen lässt sich im Uebrigen nicht, dass das Patent-Schnellfilter gröbere Verunreinigungen des Wassers vollständig zu beseitigen vermag und wird das Filter daher in solchen Fällen, in donen es sich um die Beseitigung voluninöser, flockiger Ausscheidungen, wie Eisenoxydhydrat etc., landelt, mit bestem Erfolge Verwendung finden können.

Die Handhabung des Patent-Schnellfilters ist eine ziemlich einfache und die Reinigung desselben leicht unl schnell za bewirken. Die recht beträchtliche quantitative Ergiebigkeit sowio die Abnahme diesor Ergiebigkeit bei anhaltendem Gebrauch des Filters sind von der Qualität des zu filtrirendon Wassers abhängig.

\section{Versuche mit dem Bühring'schen Kohlefilter.}

$\mathrm{Zu}$ diesen Versuchen wurde ein in oiner Privatküche angebrachtes, mit der Wasserleitung verbundenes Filter benutzt. Es konnte daher auch nur die Wirkung des Filters gegenüber dem Leitungswasser beobachtet werden, doch haben die hierbei erhaltenen Versuchsdaten hinreichende Anhaltspunkte für die Beurtheilung des Apparates ergeben.

Wie schon oben erwähnt, ist das hiosige Leitungswasser in der Regel schwach grünlich gefärbt und nicht ganz frei von suspendirten Fäsorchen und wenn es auch im allgemeinen ziemlich klar erscheint, so besitzt das Wassor doch, nachdem es durch das Kohlefilter filtrirt worden ist, unverkennbar einen grösseren Grad von Klarheit, die vorher suspendirten Fäserchen sind verschwunden und cin grtunlicher Farbton des Wassers ist selbst in stärkerer Schicht nicht mehr zu bemerken. Die hiesigen Verkäufer der Kohlefilter pflegen die Filtermasse nach 6 Monaten zu erneuern. Wie die nachstehenden Versuchsreihen ergeben, erstreckten sich die Beobachtungen bei Füllung $b$ auf die Zeitdauer von circa 7 Monaten. Trotz dieser, die normale Gebrauchszeit überschreitenden Dauer der Benutzung des Filters und trotzdem das Filter, infolge vollständiger Verschmutzung desselben durch die aus dem Leitungswasser aufgenommenen Vorunreinigungen, nach dieser Zeit eine so 
geringe quantitative Ergiebigkeit zeigte, dass aus diesem Grunde zur Erneuerung der Filtermasse geschritten werden musste, so konnte doch qualitativ eine Abnahme des unmittelbar nach der Neubeschickung beobachteten vorzüglichen mechanischen Reinigungsvermögens nicht constatirt werden. - Eine weniger günstige Wirkung kommt dem Kohlefilter hinsichtlich der Reinigung des Wassers von den darin enthaltenen Mikroorganismen zu; die einschlägigen Versuche bestätigen vielmehr, dass der Gehalt an diesen Lebewesen durch das Filter eine Vermehrung zu erfahren pflegt und zwar zeigte sich diese Vermehrung ganz besonders stark in den ersten Tagen nach der Neubeschickung des Apparates, während bei allen späteren Beobachtungen wider Erwarten zwischen nicht filtrirtem und filtrirtem Wasser keine grösseren Differenzen zu Tage traten, als dieselben bei Brunnenwässern häufig beobachtet werden können. - Die bezüglichen Versuchsreihen lasse ich zunächst folgen. (Siehe Seite 397).

Dass die Kohlefilter des Reductionsvermőgen des Wassers gegen Kaliumpermanganat und damit auch den Gehalt an gelosten organischen Verunreinigungen herabsetzen, ist eine bekannte Thatsache, die in vorstehenden Versuchsreihen ihre Bestätigung findet. Dagegen lassen obige Daten die allgemeine Annahme, dass bei längerem Gebrauch eines Kohlefilters die sich in demselben ansammelnden organischen Materien sowohl eine Vermehrung der gelösten organischen Verunreinigungen als anch eine besonders starke Zunahme des Gehalts an Mikroorganismen in dem Filtrat verursachen müssten, als nicht zutreffend erscheinen, denn trotz dor ca. siebenmonatlicheu Dauer der 2. Versuchsperiode war bei Abschluss dieser Versuchsreihe der Gehalt des filtrirten Wassers an Mikroorganismen kein aussergewöhnlich hoher, während gleichzeitig noch ein ziemlich erheblicher Minderverbrauch an Kaliumpermanganat gefunden wurde. Auffallend bleiben die beträchtlichen Schwankungen in der Abnahme des Verbrauchs an Kaliumpermanganat. Ob diese Schwankungen ihren Grund ausschliesslich in der langsameren resp. schnelleren Filtration, oder aber auch zum Theil in der Verschiedenartigkeit der gelösten organischen Verunreinigungen finden, muss ich zur Zeit dahingestellt sein lassen. Von untergeordneterer Bedeutung für hygieinische Zwecke ist die durch das Kohlefilter gleichfalls bewirkte schwache Verringerung der Härte des Wassers. Eine anderweite chemische Veränderung des Wassers wurde bei obigen Versuchen nicht beobachtot. 


\begin{tabular}{c|c|c|c|c}
\hline & $\begin{array}{c}\text { Datum } \\
\text { der } \\
\text { Untersuchung }\end{array}$ & $\begin{array}{c}\text { Varbrauch an } \\
\text { Kaliumperman- } \\
\text { ganat auf } \\
10000 \text { Theile } \\
\text { berechnet }\end{array}$ & $\begin{array}{c}\text { Verminderung } \\
\text { des Verbrauchs } \\
\text { an Kaliumper- } \\
\text { manganat } \\
\text { durch die Fil- } \\
\text { tration in \% } \%\end{array}$ & $\begin{array}{c}\text { Zur Entwicke- } \\
\text { lung auf } \\
\text { Nährgelatine } \\
\text { gelangten pro } \\
\text { ccm Wasser }\end{array}$ \\
\hline 1. & 2. & 3. & 4. & 5. \\
\hline
\end{tabular}

Fullung a, Anfang October 1884 bewirkt.

\begin{tabular}{|c|c|c|c|c|}
\hline $\begin{array}{l}\text { Leitungswasser } \\
\text { dasselbe filtrirt }\end{array}$ & $8 . / 10.1884$ & $\begin{array}{l}0,85 \\
0,51\end{array}$ & $4 \overline{0} \%$ & $\begin{array}{r}500 \\
13650\end{array}$ \\
\hline $\begin{array}{l}\text { Leitungswasser } \\
\text { dasselbe filtrirt }\end{array}$ & $22 . / 10.1884$ & $\begin{array}{l}0,79 \\
0,56\end{array}$ & $29, \overline{1} \%$ & $\begin{array}{l}150 \\
300\end{array}$ \\
\hline $\begin{array}{l}\text { Leitungswasser } \\
\text { dasselbe filtrirt }\end{array}$ & $5 . / 11.1884$ & $\begin{array}{l}1,23 \\
0,85\end{array}$ & $30, \overline{9} \%$ & $\begin{array}{l}140 \\
300\end{array}$ \\
\hline $\begin{array}{l}\text { Leitungswasser } \\
\text { dasselbe filtrirt }\end{array}$ & $5 . / 12.1884$ & $\begin{array}{l}0,90 \\
0,43\end{array}$ & $52, \overline{2} \%$ & $\begin{array}{l}240 \\
910\end{array}$ \\
\hline
\end{tabular}

Füllung b, Anfang April 1885 bewirkt.

\begin{tabular}{|c|c|c|c|c|}
\hline $\begin{array}{l}\text { Leitungswasser } \\
\text { dasselbe filtrirt }\end{array}$ & $21 . / 4.1885$ & $\begin{array}{l}1,64 \\
0,95\end{array}$ & $42 \overline{\%}$ & $\begin{array}{r}62 \\
126\end{array}$ \\
\hline $\begin{array}{l}\text { Leitungswasser } \\
\text { dasselbe filtrirt }\end{array}$ & $18 . / 5.1885$ & $\begin{array}{l}1,55 \\
0,75\end{array}$ & $51, \overline{6} \%$ & $\begin{array}{l}140 \\
725\end{array}$ \\
\hline $\begin{array}{l}\text { Leitungswasser } \\
\text { dasselbe filtrirt }\end{array}$ & $9 . / 6.1885$ & $\begin{array}{l}1,71 \\
0,96\end{array}$ & $43, \overline{8} \%$ & $\begin{array}{l}450 \\
530\end{array}$ \\
\hline $\begin{array}{l}\text { Leitungswasser } \\
\text { dasselbe filtrirt }\end{array}$ & $19 . / 9.1885$ & $\begin{array}{l}1,11 \\
0,79\end{array}$ & $28, \overline{8} \%$ & $\begin{array}{l}240 \\
550\end{array}$ \\
\hline $\begin{array}{l}\text { Loitungswasser } \\
\text { dasselbe filtrirt }\end{array}$ & $10 . / 11.1885$ & $\begin{array}{l}1,48 \\
0,98\end{array}$ & $33, \overline{7} \%$ & $\begin{array}{l}540 \\
780\end{array}$ \\
\hline
\end{tabular}

Fūllung c, am 20. December 1885 bewirkt.

\begin{tabular}{|c|c|c|c|c|}
\hline $\begin{array}{l}\text { Loitungswasser } \\
\text { dasselbe filtrirt }\end{array}$ & $21 . / 12.1885$ & $\begin{array}{l}1,74 \\
0,90\end{array}$ & $48, \overline{2 \%}$ & $\begin{array}{r}420 \\
16500\end{array}$ \\
\hline $\begin{array}{l}\text { Leitungswasser } \\
\text { dasselbe filtrirt }\end{array}$ & $12 . / 2,1885$ & $\begin{array}{l}1,52 \\
1,32\end{array}$ & $13, \overline{1} \%$ & $\begin{array}{l}20 \\
68\end{array}$ \\
\hline $\begin{array}{l}\text { Leitungswasser } \\
\text { dasselbe filtrirt }\end{array}$ & 5./3. 1886 & $\begin{array}{l}1,27 \\
0,79\end{array}$ & $37, \overline{8} \%$ & 75 \\
\hline
\end{tabular}

Wie bereits oben erwähnt worden ist, konnten Versuche mit anderem, stärker verunreinigten Wasser nicht angestellt werden. Nach den vorstehenden Beobachtungen dürite es indess kaum zu bezweifeln sein, dass das Kohlefilter auch unter wesentlich ungünstigeren Vorbedingungen in qualitativer Beziehung befriedigend functioniren wird. 
Die Handhabung des Kohlefilters ist eine sehr einfache, die quantitative Leistung desselben befriedigend, selbstverständlich indess ebenso wie die Zeitdauer der Brauchbarkeit der Filtermasse von der mehr oder weniger starken Verunreinigung des zu filtrirenden Wassers abhängig. Eine Reinigung der Filtermasse ist nicht ausführbar, sondern ev. eine Neubeschickung erforderlich.

\section{Versuche mit dem Mikromembranfilter.}

$\mathrm{Zu}$ der Construirung dieses Filtrirapparates ist, nach der schon oben erwähnten Breyer'schen Brochüre, der Erfinder desselben durch die Erwägung veranlasst, dass die Hauptursache der vielfach constatirten schädlichen Wirkung mancher Wässer dem Gehalt des Wassers an pathogenen Keimen zuzuschreiben sei und dass demgemäss dio Versuche, das Wasser zu verbessern, ihren Schwerpunkt in der Beseitigung der Mikroorganismen finden müssten. Diesen Zweck glanbte Breyer durch Anwendung einer höchst feinfaserigen Filtermasse, deren Filteroffnungen kleiner seien als die Mikroorganismen, erreichen zu können. Vergleichende Beobachtungen lieferten das Frgebniss, dass die feinsten von der Natur dargebotenen und zu Filtrirzwecken verwendbaren Fasern, wie Baumwolle, Badeschwamm etc., infolge der grossen, die Mikroorganismen ohne Schwierigkeit durchlassenden Poren, eine bakteriendichte Filtermasse nicht zu liefern vermöchten. Ein recht glücklicher Griff liess Breyer im Asbest ein Material finden, welches durch geeignete Behandlung in solchem Maasso zerkleinert werden kann, dass die über einander abgelagerten Fäserchen ein hüchst zartes Netz bilden, dessen Oeffnungen, wie Bre yer auf Grund der von ihm vorgenommenen Messungen der Mikrodimensionen sowohl dieser Filteroffnungen als auch verschiedener pathogener Bakterienarten angiebt, so klein sein sollen, dass die Mikroorganismen dieselben nicht zu passiren vermoggen. Den Beweis für die Richtigkeit dieser Angabe sucht der Erfinder des Filters durch indirecte und directe Versuche beizubringen. Zunächst betont er, dass die in natürlichen Wässern ab und zu vorkommenden Thontrübungen, welche in der Regel selbst ron grossen Sandfiltern nicht zurückgehalten werden, durch das Mikromembranfilter vollatändig beseitigt werden können. Da nun die Thonpartikelchen zum Theil eine geringere Grösse besitzen, als die Mikroorganismen, so folgert Breyer, dass das Mikromembranfilter daher auch alle Mikroorganismen bei der Filtration ausscheiden müsse. Weiter führt 
Breyer aus, dass verschiedene Farbstoffe, die weitaus kleinkörniger seien als Thonerde, wie Ultramarinblau, Cochenilleroth und Anilinblau, durch das Filter zurückgehalten werden und schliesslich haben Filtrirversuche mit Milzbrandblut zu dem Resultat geführt, dass Milzbrandsporen und Bacillen im Filtrat nicht nachgewiesen werden konnten, während dagegen bei der Filtration von tuberkulossem Sputum das Filtrat bei Meerschweinchen noch geringgradige Tuberkulose hervorrief. Letztere Wirkung sucht Breyer durch die Annahme zu erklären, dass nicht Tuberkelbacillen, sondern nur Sporen derselben durch das Filter hindurchgegangen seien. Letzterer Versuch lässt es nun wohl mindestens zweifelhaft, ob nicht trotzdem auch Bacillen die Filtermembran passirt haben, was im Uebrigen nach dem Ausfall meiner weiter unten $z u$ besprechenden Versuche nicht unwahrscheinlich ist.

Zu den Versuchen mit dem Mikromembranfilter wurde ein aus 10 Doppelelementen bostehendes Filter (Type II, Motiv. C) sowie ausserdem ein mir von dem Erfinder des Filters in liebenswurdigster Weise zur Verfügung gestelltes "Taschenfilter " benutzt. Die Versuche, die mechanische Reinigung des Wassers durch den Filtrirapparat betreffend, ergaben, dass Leitungswasser nach dem Filtriren unter Beseitigung der suspendirten Fäserchen und des schwach grünlichen Farbtons völlig klar geworden war; ferner wurde stark getrübtes und grüngelblich gefärbtes Oderwasser durch die Filtration gleichfalls absolut klar und farblos erhalten und ebenso führte ein weiterer Versuch mit durch Thonzusatz gotrubtem Wasser zu dem Resultat, dass die Thonpartikelchen von dem Filter vollständig zurückgehalten wurden, allerdings unter wesentlicher Verminderung der quantitativen Ergiebigkeit.

Gleichzeitig mit vorstehenden Beobachtungen wurden Ermittelungen über die Verminderung der im Wasser vorhandenen Mikroorganismen angestellt. Das zu diesen Untersuchungen benutzto Oderwasser hat, da die Versuche nur die Feststellung der Abnahme der Zahl der Bakterien bezweckten, in der Regel einige Tage im Untersuchungslokale gestanden; der Befund an Mikroorganismen überschreitet daher den normalen Gehalt des Oderwassers ganz erheblich.

In den hier folgenden Versuchsreihen ist der Gehalt an zur Entwickelung gelangten Mikroorganismen pro ccm Wasser angegeben. 
A. Link, Wasserfiltrirapparate.

Anzahl der Mikroorganismen

vor und nach

$\begin{array}{rcccrr}\text { a. } & \text { Oderwasser } & \text {. } & \text {. } & 189000 & 1700 \\ \text { b. } & \text { do. } & . & . & 235000 & 8600 \\ \text { c. } & \text { do. } & . & . & 63000 & 3900 \\ \text { d. } & \text { Leitungswasser } & . & 585 & 300 \\ \text { e. } & \text { Oderwasser } & \text {. } & \text {. } & 58500 & 900 \\ \text { f. } & \text { Leitungswasser } & . & 150 & 50 \\ \text { g. } & \text { Oderwasser . } & . & 13200 & 1630 \\ \text { h. } & \text { Brunnenwasser } & . & 28400 & 2080 \\ \text { i. } & \text { Leitungswasser } & . & 50 & 4 \\ \text { k. } & \text { Brunnenwasser } & . & 130 & \mathbf{1 5} \\ \text { l. } & \text { do. } & . & 300 & 8 \\ \text { m. } & \text { do. } & . & 129600 & 5400\end{array}$

$\mathrm{Zu}$ den unter $\mathrm{d}-\mathrm{m}$ aufgeführten Versuchen wurde das jeweilig vorher sterilisirte Taschenfilter benutzt.

Vorstehende Zahlenreihen geben einen guten Beweis dafür, dass das Mikromembranfilter bezïglich Verminderung der Mikroorganismen so Vorzagliches leistet, wie bisher keiner der bekannteren Filtrirapparate, dagegen wird die absolute Reinigung des Wassers in dieser Beziehung wie solche nach den früheren Ausfuhrungen dem Apparate von dem Erfinder desselben zugeschrieben wird, durch das Filter zur Zeit noch nicht bewirkt. Eine Wirkung des Filters in chemischer Beziehung, wird von dem Erfinder für dasselbe nicht beansprucht. Einschlägige Versuche ergaben, dass zwar bei Anwendung ganz neuer Filterlamellen eine Verminderung der organischen Substanzen resp. des Reductionsvermögens gegen Kaliumpermanganat eintrat, dass aber diese Wirkung nach einiger Zeit nicht mehr zu bemerken war. Eine Veränderuug der anderweiten Componenten des Wassers trat bei den Beobachtungen nicht zu Tage.

Nach den bei vorstehenden Versuchen erhaltenen Resultaten erfullt das Mikromembranfilter den vom Erfinder ins Auge gefassten Zweck, die mechanische Reinigung sowie die Befreiung des Wassers von seinem Gehalt an Mikroorganismen, in sehr beachtenswerther Weise. Wenn die Wirkung des Apparates in letzterer Beziehung zur Zeit noch keine vollkommene ist, so wird dies vielleicht darin zu suchen sein, dass der Erfinder des Filters bei der Berechnung und Feststellung der Grössenverhältnisse der ziemlich erheblichen Eigen- 
bewegung zahlreicher Bakterienarten, welche es denselben ermöglichen dürfte, verhältnissmässig kleine Hohlräume zu passiren, nicht hinreichend Rechnung getragen hat. Moglicherweise wird schon eine etwas weitergehende Verdickung der Filterlamellen das gewünschte Ziel erreichen lassen. Da die Herstellung eines die Mikroorganismen mit Sicherheit völlig ausscheidenden Wasserfilters für hygieinische Zwecke zweifelsohne von grosser Bedeutung ist, so steht zu hoffen, dass der mit grossem Scharfsinn construirte Apparat baldigst einer weiteren Vervollkommnung entgegengeführt wird.

Im Gegensatz zu dem Patent-Schnellfilter und dem Kohlefilter ist das Mikromembranfilter ziemlich complicirt zusammengesetzt. Die verhältnissmässig grossen und dabei gegen irgend welche Unregelmässigkeiten des Betriebes recht empfindlichen Filterlamellen bedingen eine sehr sorgfältige Handhabung des Apparates. Bei etwaigem Unbrauchbarwerden der Filterlamellen, infolge starker Verschmutzung oder aber Zerreissen derselben, ist der Belag mit neuen Lamellen ohne besondere Schwierigkeit durchführbar.

Auch bei diesem Apparat ist die quantitative Leistungsfähigkeit, sowie die Abnahme derselben von der Beschaffenheit des zu filtrirenden Wassers nicht unabhängig.

Nicht uninteressant dürften im Anschluss an obige Mittheilungen einige Notizen über das Oderwasser und das aus diesem gewonnene hiosige Leitungswasser erscheinen.

Wie bereits vorstehend mehrfach erwähnt ist, enthält das Wasser der Oder starke Trübungen verschiedenartigen Ursprungs, es besitzt einen grünlichen Farbton, ist schwach ammoniakhaltig und zeigt ein je nach der Jahreszeit und Witterung schwankendes Reductionsvermögen gegen Kaliumpermanganat. In den Wasserwerken der hiesigen Wasserleitung wird das Oderwasser durch grosse Sandfilter filtrirt. Das filtrirte Wasser ist im allgemeinen ziemlich klar, indess in der Regel nicht ganz frei von suspendirten Fäserchen, der grünliche Farbton des Wassers ist wesentlich vermindert, der Ammoniakgehalt verschwindet entweder ganz oder bis auf geringe Spuren und das Reductionsvermögen gegen Kaliumpermanganat wird ermässigt. Ganz besonders beachtenswerth aber ist die Leistung der Sandfilter beztuglich Verminderung des Gehalts an Mikroorganismen, welche Leistung sich in nachstehenden Zahlenreihen ziffermalssig ausgedruckt findet. 
Zur Entwickelung auf Nahrgelatine gelangten pro ccm Wasser

Beobachtungszeit

8./10. 1884

$22 . / 10 . \quad 1884$

18./5. $\quad 1885$

8.16 .

$9 . / 6$.

19./9.

10.111.
Oderwasser (bei dem Leitungswasser (der LeiGarnison-Lazareth tung im Garnison- Lazageschöpft)

15000 reth entnommen)

10300

4500

10800

13400

11700

5240
500

150

140

585

450

240

540

Die weiten Entfernungen gestatteten mir leider nicht, das zu diesen Untersuchungen benutzte Oderwasser oberhalb der Stadt in der Nähe der Wasserwerke zu entnehmen. Die etwaigen Differenzen gegenüber dem in der Stadt geschopften Oderwasser werden indess voraussichtlich nicht sonderlich gross sein und jedenfalls nicht an die in obiger Zusammenstellung hervortretenden Schwankungen im Gehalt des Oderwassers an Mikroorganismen heranreichen. Andererseits würde unmittelbar nach dem Passiren der Sandfilter entnommenes Wssserleitungswasser sicher ein noch gunstigeres Resultat liefern, als das aus einer Ausflussstelle in der Stadt erhaltene Wasser, da bekanntlich innerhalb der Reservoire und des Röhrennetzes eine nicht unbeträchtliche Vermehrung der durch die Filtration nicht ganz beseitigten Mikroorganismen stattzufinden pflegt. Jedenfalls lassen die von den kleineren Filtrirapparaten bisher nicht erreichte Wirkungen des Sandfilters bezüglich Verminderung des Gehalts an Mikroorganismen, sowie die recht beträchtliche mechanische und chemische Verbesserung des Wassers das Sandfilter den vorbesprochenen Filtrirapparaten, trotzdem diese manchmal in einzelnen Richtungen mehr zu leisten vermögen, in seiner Gesammtleistung überlegen erscheinen.

Stettin, Anfang März 1886.

\section{B. Monatsberieht.}

\section{Pharmacentische Chemie.}

Atropinum santonjcum. - F. Bombel on versuchte mit Santonsäure eine Atropinverbindung herzustellen und hat guten Erfolg erzielt. Die Santonsäure ist in minimaler Menge ein Antisepticum und lässt teine Pilze aufkommen; sie ist reizlos, so dass eine gesättigte wässrige Lösung ins Auge 